\title{
From TORTORA to MegaTORTORA—Results and Prospects of Search for Fast Optical Transients
}

\author{
Grigory Beskin, ${ }^{1}$ Sergey Bondar, ${ }^{2}$ Sergey Karpov, ${ }^{1}$ Vladimir Plokhotnichenko, ${ }^{1}$ \\ Adriano Guarnieri, ${ }^{3}$ Corrado Bartolini, ${ }^{3}$ Giuseppe Greco, ${ }^{3}$ Adalberto Piccioni, ${ }^{3}$ \\ and Andrew Shearer ${ }^{4}$
}

${ }^{1}$ Special Astrophysical Observatory of Russian Academy of Sciences, Nizhnij Arkhys 369167, Russia

${ }^{2}$ Arkhyz Branch, Institute for Precise Instrumentation, Nizhnij Arkhys 369167, Russia

${ }^{3}$ Astronomy Department, Bologna University, 40126 Bologna, Italy

${ }^{4}$ School of Physics, National University of Ireland, University Road, Galway, Ireland

Correspondence should be addressed to Grigory Beskin, beskin@sao.ru

Received 28 June 2009; Accepted 12 January 2010

Academic Editor: Joshua S. Bloom

Copyright (c) 2010 Grigory Beskin et al. This is an open access article distributed under the Creative Commons Attribution License, which permits unrestricted use, distribution, and reproduction in any medium, provided the original work is properly cited.

\begin{abstract}
To study short stochastic optical flares of different objects (GRBs, SNs, etc.) of unknown localizations as well as NEOs it is necessary to monitor large regions of sky with high-time resolution. We developed a system consisting of widefield camera with field of view of 400-600 sq.deg. which uses TV-CCD with $0.13 \mathrm{~s}$ temporal resolution to record and classify optical transients, and a fast robotic telescope aimed to perform their spectroscopic and photometric investigation just after detection. Such two-telescope complex, combining wide-field camera TORTORA and robotic telescope REM, operated from May 2006 at La Silla ESO observatory. Some results of its operation, including first high time resolution study of optical transient accompanying GRB and discovery of its fine time structure, are presented. Also, prospects for improving the efficiency of such observations are given, and a project of a next generation wide field monitoring system, the MegaTORTORA, is described.
\end{abstract}

\section{Introduction}

The efforts for searching and investigation of optical flashes accompanying the Gamma-Ray Bursts were somewhat contradictory from the beginning. The large amount of bursts shorter than 2 seconds (nearly 30\%) and the presence of fine temporal structure, down to milliseconds, in their light curves [1] obviously require both continuous optical monitoring of space telescopes fields of view and application of detectors with high temporal resolution [2-4]. But in spite of that until 2002 nearly all studies of GRB optical emission have been performed in a follow-up regime, by pointing a telescope towards burst positions measured by a satellite, and observing with exposures larger than 10 seconds. However, even for the best possible manners of coordinate messages distribution [5], such observations start at least 5-30 seconds after the burst onset and are unable to study the burst temporal structure with resolution comparable to ones available in gamma-ray band [6-9]. Obviously, optical flashes accompanying short gamma-ray bursts cannot be detected in such a way, while for long bursts it is impossible to compare optical and gamma-ray light curves. Even later, when wide-field monitoring cameras able to detect optical transients independently from satellite triggers, like WIDGET [10], RAPTOR [11], BOOTES [12, 13], and $\pi$ of the Sky [14], appeared, their low-temporal resolution significantly limited the possibilities of investigating the physical nature of the bursts, and especially - their central engines. Indeed, presently it is widely accepted that GRB central engines are compact relativistic objects-isolated or binary neutron stars [15] or stellar mass black holes $[16,17]$ interacting with massive accretion disks. Observations in different wavebands with temporal resolution close to time scales of nonstationary processes near the event horizon, from milliseconds to seconds, may be crucial for understanding the physics of such objects. 


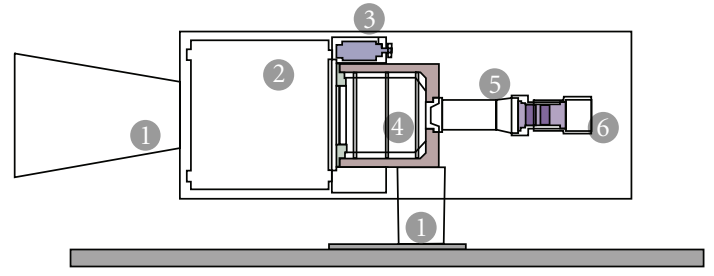

Figure 1: Schematic view of a TORTORA design. (1) Protective blend, (2) main objective, (3) main objective focusing unit, (4) image intensifier, (5) transmission optics and CCD focusing unit, and (6) fast CCD.

For that purpose, since late 1990s we are developing the strategy of optical monitoring with high temporal resolution in the wide fields comparable to ones of spaceborne telescopes. Initially it has been proposed $[18,19]$ to use instruments with large mirrors of relatively low quality, air Cerenkov telescopes, or solar concentrators, with phototubes able to operate with temporal resolution down to microseconds. Later, however, we finished the design of a wide-field camera equipped with image intensifier and a fast low-noise CCD. The prototype of such a camera, FAst Variability Optical Registrator (FAVOR), is operated since 2003 near Russian 6-m telescope [2, 20], while similar Telescopio Ottimizzato per la Ricerca dei Transienti Ottici RApidi (TORTORA) is mounted since 2006 on top of Italian REM telescope [21] in La-Silla Observatory (ESO, Chile) as a part of a TORTOREM [22] two-telescope complex [23]. The latter camera has detected and studied with unprecedented temporal resolution the optical emission of a Naked-Eye Burst [24-26].

Here we describe the design and implementation of TORTORA camera, present some results of its operation, and propose the project of a next generation wide field monitoring system, the MegaTORTORA, able to catch much fainter transients in a wider field of view, and acquire complete multicolor and polarimetric information on them.

\section{Design of TORTORA Wide-Field Camera}

Parameters of FAVOR and TORTORA cameras in comparison to other wide-field monitoring systems currently in operation are presented in Table 1. The only cameras combining both wide field of view and relatively high-time resolution are ones presented here.

The schematic view of TORTORA is shown in Figure 1, its technical parameters is listed in Table 2, and the camera photo in Figure 2. The camera consists of the main objective (2), its focusing unit (3), the image intensifier (4) used to downscale and amplify the image, transmission optics (5), and the fast low-noise TV-CCD (6). TORTORA is installed on top of REM robotic telescope, which has alt-azimuthal mounting.

Fast TV-CCD matrix operates at 7.5 frames per second regime with $0.128 \mathrm{~s}$ time exposure and gaps between frames negligibly small. The data from CCD is broadcasted through the local gigabit Ethernet network to one terabyte storage
TABLE 1: Typical wide-field monitoring cameras currently in operation. For FAVOR and TORTORA the limits correspond to $3 \sigma$ detection on a single frame and differ from their real-time operational values.

\begin{tabular}{lccc}
\hline Name & $\begin{array}{c}\text { Field of view } \\
\text { (degrees) }\end{array}$ & $\begin{array}{c}\text { Temporal } \\
\text { resolution } \\
\text { (seconds) }\end{array}$ & Limit \\
\hline WIDGET & $62 \times 62$ & 5 & $10^{\mathrm{m}}$ \\
RAPTOR A/B & $40 \times 40$ & 60 & $12^{\mathrm{m}}$ \\
RAPTOR Q & $180 \times 180$ & 10 & $10^{\mathrm{m}}$ \\
BOOTES & $16 \times 11$ & 30 & $12^{\mathrm{m}}$ \\
BOOTES-AllSky & $180 \times 180$ & 30 & $10^{\mathrm{m}}$ \\
$\pi$ of the Sky & $33 \times 33$ & 10 & $11.5^{\mathrm{m}}$ \\
AROMA-W & $25 \times 35$ & $5-100$ & $10.5^{\mathrm{m}}-13^{\mathrm{m}}$ \\
MASTER-VWF & $20 \times 21$ & 5 & $11.5^{\mathrm{m}}$ \\
MASTER-Net & $30 \times 30$ & 1 & $9^{\mathrm{m}}$ \\
\hline FAVOR & $16 \times 24$ & 0.13 & $10^{\mathrm{m}}-11.5^{\mathrm{m}}$ \\
TORTORA & $24 \times 32$ & 0.13 & $9^{\mathrm{m}}-10.5^{\mathrm{m}}$ \\
\hline
\end{tabular}

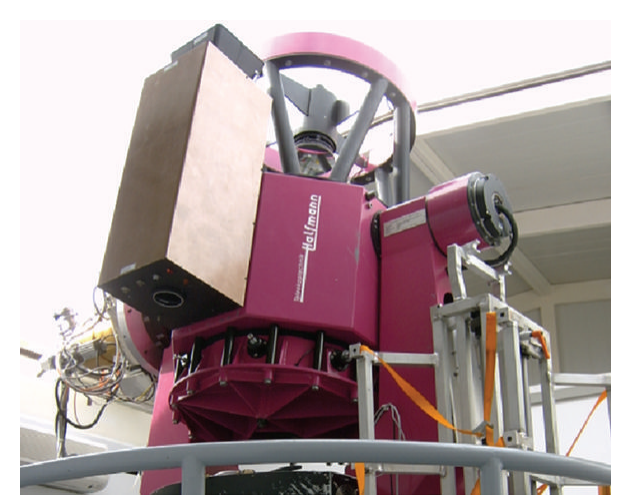

Figure 2: Photo of a TORTORA camera, mounted on top of REM robotic telescope in La-Silla Observatory, ESO, Chile.

RAID array. The data flow rate for the system is about $20 \mathrm{Mb} / \mathrm{s}$, and so the storage may keep the raw data only for one or two days.

Also, the raw data are transmitted to the real-time processing PC operating the custom pipeline software under Linux OS. The pipeline performs the detection and classification of transient events of various types and tries to recognize already known objects, by comparing the time and position of each events with catalogues of satellites and with star catalogue to minimize the number of false events due to stellar scintillations under bad weather conditions.

\section{Detection Methodology}

Wide-field monitoring cameras with high temporal resolution may be used for detection and investigation of various classes of transient events - variable stars, supernovae, active galactic nuclei, MACHOs, planetary transits over starswith fixed, but unknown a priori coordinates. On the other hand, cameras like FAVOR or TORTORA may detect also the 
TABLE 2: Technical parameters of TORTORA camera.

\begin{tabular}{lclclc}
\hline \multicolumn{2}{c}{ Main objective } & \multicolumn{2}{c}{ Intensifier } & & CCD \\
\hline Diameter & $120 \mathrm{~mm}$ & Photocathode & S20 & Model & VS-CTT285-2001 \\
Focus & $150 \mathrm{~mm}$ & Diameter & $90 \mathrm{~mm}$ & Dimensions & $1388 \times 1036 \mathrm{pix}$ \\
$\mathrm{D} / \mathrm{F}$ & $1 / 1.2$ & Gain & 150 & Image scale & $81^{\prime \prime} / \mathrm{pix}$ \\
Field of view & $24 \times 32 \mathrm{deg}$ & Scaling coefficient & 7.7 & Exposures & $0.13-10 \mathrm{sec}$ \\
& & Quantum efficiency & $10 \%$ & Pixel size & $6.45 \mu \mathrm{m}$ \\
\hline
\end{tabular}

moving objects—satellites, space debris, comets, asteroids, meteors. For TORTORA, we developed special algorithms able to detect both classes of transients.

Due to very high data flow from the camera, it is impossible to use standard image reduction packages, so we have developed a fast transient detection algorithm based on the "differential imaging" method [27], which implies statistical analysis of temporal behaviour of each pixel over $N=100$ previous frames, that is, 13 seconds. The current value of the pixel $I$ is being compared with the running mean $\langle I\rangle=\sum I / N$ and standard deviation $\sigma_{I}=$ $\sqrt{\left(\sum I^{2}-\left(\sum I\right)^{2} / N\right) /(N-1)}$, and the significance of excess over the mean is computed as $A=(I-\langle I\rangle) / \sigma_{I}$. Then, all the pixels with deviations over the mean of $3 \sigma$ and greater are clustered into extended objects. Some objects, like singlepixel ones, are filtered out as they are most likely due to noise.

After the extraction of objects from current frame, the reduction pipeline compares their positions with trajectories of transients seen on previous ones (all objects here are assumed to be moving, but some of them-with zero velocity). Detection of object on three successive frames (in half a second) is enough to classify it into one of three possible classes- "noise," if the object disappears, moving event, if it has statistically significant motion, or stationary transient. The case of slowly moving geostationary satellites is handled by comparing the event position with regularly updated satellite catalogue [28].

Detection of meteors, however, requires a different approach, as most of them may be seen on single or two successive frames only. Also, their motion is significantly faster than that one of satellites. So, the meteors are selected by geometric length and flux criteria only.

The astrometric and photometric calibration is performed regularly (once per minute in a case of TORTORA camera, as it has an alt-azimuthal mounting with rotating field of view) by means of additional SExtractor-based [29] pipe-line and custom WCS matching code based on Tycho-2 stellar catalogue [30].

So, the real-time pipeline is able to detect and classify any bright optical transient in a 0.4 second ( 3 frames) since its onset, before it hides from differential imaging algorithm. Example of such short flare is presented in Figure 3. Then, the information on the event may be sent to the robotic telescope to perform its detailed investigation. Also, all the relevant information on the transient, including its light curve, trajectory, and pieces of raw images containing it, is stored for the subsequent offline investigation and statistical analysis.
TABLE 3: Upper limits on the constant flux and sinusoidal variability of gamma-ray bursts, observed by TORTORA wide-field camera in follow-up regime.

\begin{tabular}{lllcl}
\hline \multirow{3}{*}{ Burst } & $\begin{array}{l}\text { Time since } \\
\text { event } \\
\text { (seconds) }\end{array}$ & $\begin{array}{l}\text { 12 s limit } \\
(100 \\
\text { frames })\end{array}$ & $\begin{array}{c}\text { Variability } \\
\text { timescale } \\
(\mathrm{Hz})\end{array}$ & $\begin{array}{l}\text { Variability } \\
\text { limit }\end{array}$ \\
\hline GRB060719 & 59 & 12.4 & $0.01-3.5$ & $15.3^{\mathrm{m}}$ \\
GRB061202 & 92 & 11.3 & $0.1-3.5$ & $14.0^{\mathrm{m}}$ \\
GRB060719 & 118 & 11.3 & $0.01-3.5$ & $16.4^{\mathrm{m}}$ \\
\hline
\end{tabular}

\section{TORTORA Results}

TORTORA camera operates since June 2006, approximately half of observational time (when REM is not performing its scheduled programme) it follows up regions of the sky observerd by the Swift satellite, according to real-time pointing information distributed through the GCN network [5]. The regime of follow-up observations of transients detected independently by TORTORA with the REM telescope is now in testing stage.

For each observational night, the camera detect approximately 300 meteors and 150 satellites of various brightness.

4.1. Follow-up Observations of Gamma-Ray Bursts. Due to REM telescope operation in follow-up regime in respect to Swift satellite triggers, TORTORA camera has been able to observe the regions of localization of three gamma-ray bursts in a short time since the event [31-33].

The integral data on all these follow-up observations are presented in Table 3. Stationary flux limits have been derived from 100 -frame average images ( $12.8 \mathrm{~s}$ effective exposure).

4.2. Observations of Naked-Eye Burst. March 19 and 20, 2008 became the most fruitful days for wide-field monitoring systems around the world. It brought up 5 GRBs in a row, all within 24 hours, one of which, GRB080319B [34], is the brightest ever seen in gamma-rays and optical range, and the first one to be detected by monitoring systems. Its field of view had been images before, during, and after the gamma event by "Pi of the Sky" [35], RAPTOR Q [36], and TORTORA [24] cameras.

We observed the region of GRB080319B [24, 25] since 05:46:22 UT, nearly half an hour before the burst (burst time is 06:12:49 UT), during the event and for several tens of minutes after its end. At 06:13:13 UT till 06:13:20 UT REM telescope performed automatic repointing after receiving the coordinates distributed by Swift [34], which moved 


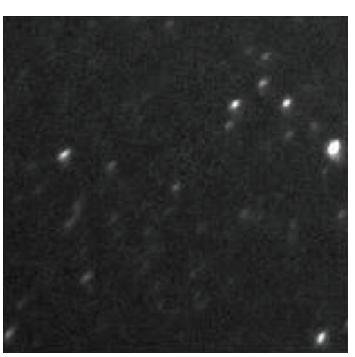

(a)

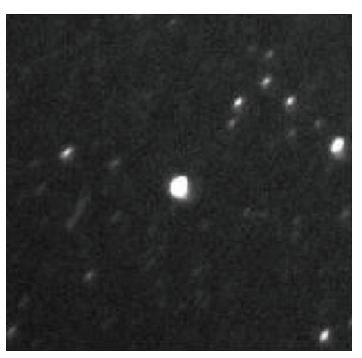

(b)

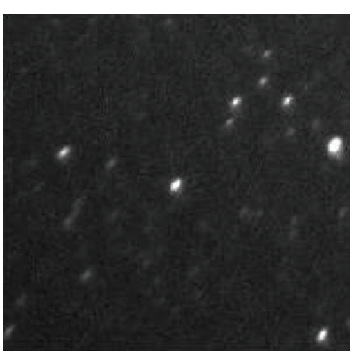

(c)

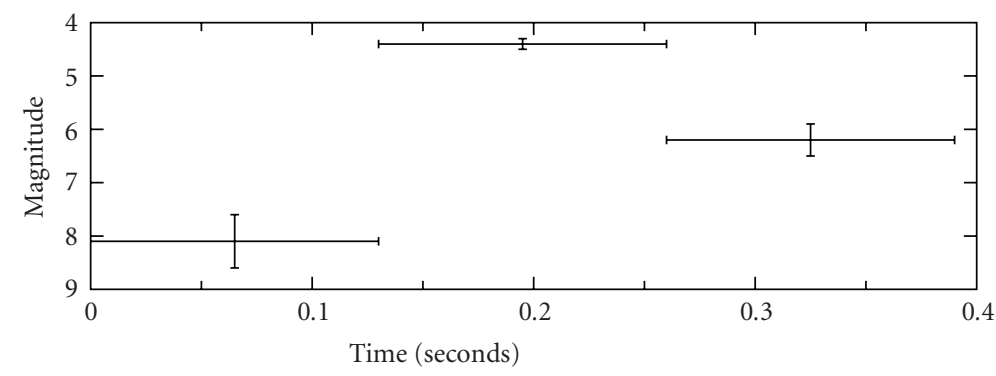

(d)

FIgure 3: Example of a short satellite flare detected by the camera. Total length of the event is 0.4 sec (seen on 3 successive frames).

the position of the burst from the edge of the TORTORA field of view towards its center. Sample images of the burst region at different stages of the event are presented in Figure 4.

The data acquired have been processed by a pipeline including TV-CCD noise subtraction, flat-fielding to compensate vignetting due to objective design, and custom aperture photometry code taking into account non-Poissonian and nonergodic pixel statistics caused by image intensifier. For the REM repointing time interval fluxes have been derived using custom elliptic aperture photometry code after summation of 10 consecutive frames ( $1.3 \mathrm{~s}$ effective exposure) with compensated motion of the stars, therefore no full-resolution measurements ( $0.13 \mathrm{~s}$ exposure) are available for this interval.

TORTORA acquired the data in white light with sensitivity defined by the S20 photocathode used in the image intensifier [2]. Instrumental object magnitudes have then been calibrated to Johnson $V$ system using several nearby Tycho-2 [30] stars. A quick-look low resolution light curve (lacking the data during the REM repointing interval) has been published $[24,25]$ and has been found to agree with results of other wide-field monitoring cameras which also observed this burst, such as "Pi of the sky" [35] and RAPTOR [36]. Our complete full resolution light curve along with the low resolution one (after the restoring of the gap) is shown in Figure 5.

We clearly detected the transient optical emission since approximately 10 seconds after the trigger. It then displayed fast $\sim t^{4}$ rise, peaked at $V \approx 5.5^{\mathrm{m}}$, demonstrated $1.5-2$ times variations on a several seconds time scale and decayed as $\sim t^{-4.6}$ until went below TORTORA detection limit at about hundred seconds since trigger. The gamma emission itself ended at 57 th second.
The light curve clearly shows four peaks with similar amplitudes, durations, and shapes. We decomposed it into four components described by a simple Kocevski [37] profile. We stress that distances between peaks are nearly the same within the errors and are around $8.5 \mathrm{~s}$ in observer frame, which corresponds to $4.4 \mathrm{~s}$ in the rest frame at $z=0.937$ [25].

Therefore, for the first time, we have a clear detection of a periodic variations of prompt optical emission on a few seconds time scale.

We then subtracted the smooth curve, formed by four fitted peaks, from the original data and studied the residuals shown in the lower panel of Figure 5. Power spectral analysis of different subintervals of the burst revealed the signature of a periodic intensity variations during the last peak, since $T+40 \mathrm{~s}$ till $T+50 \mathrm{~s}$, shown in Figure 6 . No other intervals of the light curve show any variability in $0.1-$ $3.5 \mathrm{~Hz}(0.3-10 \mathrm{~s})$ range with power exceeding $15 \%$ before and $10 \%$ after the REM repointing. To exclude artificial nature of these variations we performed analysis of each comparison star separately in the same way as of the object. Neither comparison stars nor background displays any similar periodic feature during either the whole time interval or the last peak.

The significance level of the power density spectrum feature shown in Figure 6 is approximately 1\%. The period and amplitude of the corresponding sinusoidal component, derived by means of nonlinear least squares fit, are $1.13 \mathrm{~s}$ and $9 \%$, respectively.

To compare the temporal structure of optical and gamma-ray light curves we performed the cross-correlation analysis, using the plateau phase only, excluding the first and last 12 seconds of the burst both in optical and in gamma, which are obviously highly correlated [38] (see Figure 7). The correlation between the full-resolution optical 


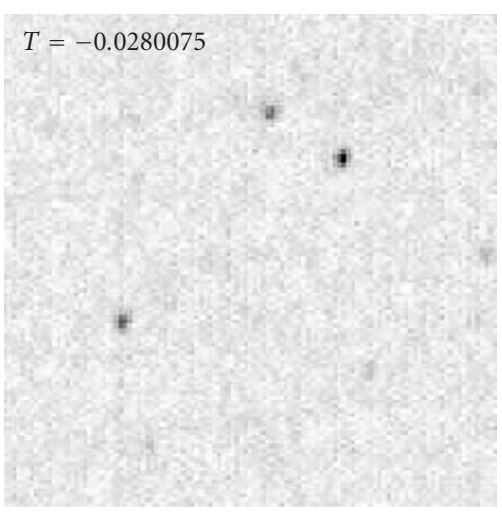

(a)

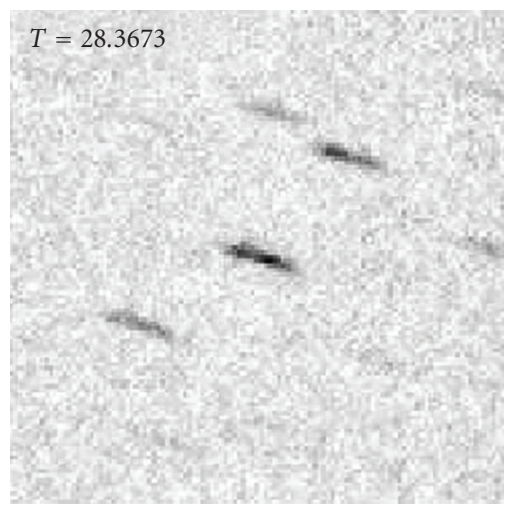

(d)

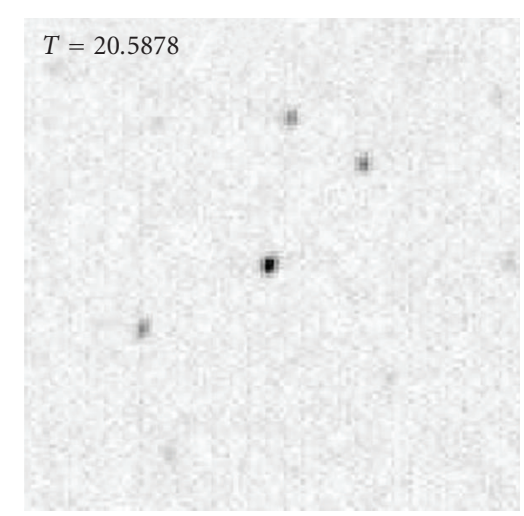

(b)

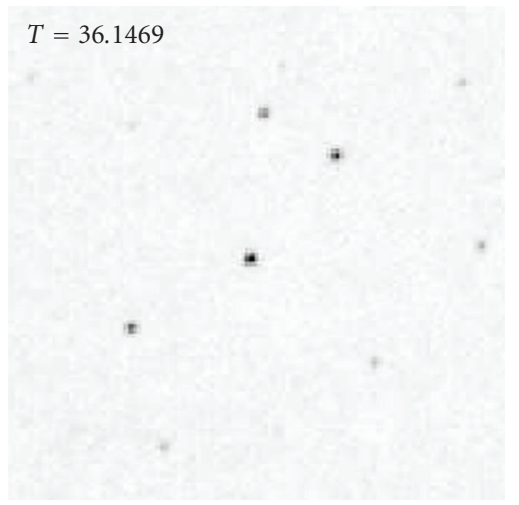

(e)

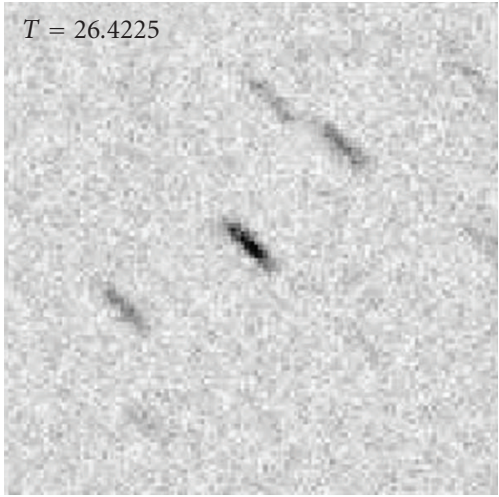

(c)

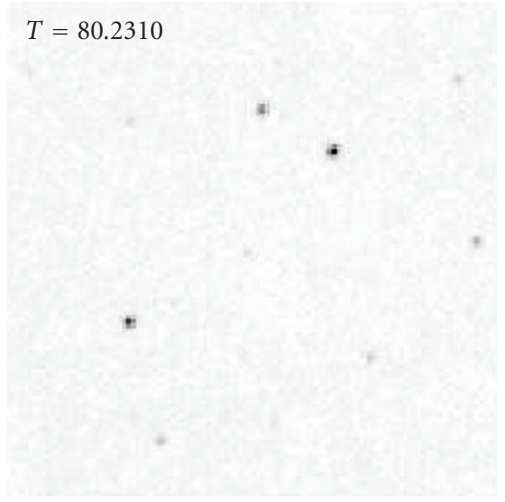

(f)

FIgURE 4: The development of prompt optical emission from GRB080319b as seen by TORTORA camera. Sums of 10 consecutive frames with $1.3 \mathrm{~s}$ effective exposures are shown for the gamma-ray trigger time $(T=0 \mathrm{~s})$, the maximum brightness time during the first peak $(T=20.5 \mathrm{~s})$, two middle-part moments $(T=26.4 \mathrm{~s}$ and $T=28.4 \mathrm{~s})$, at the last peak $(T=36 \mathrm{~s})$, and during early afterglow $(T=80 \mathrm{~s})$ stages. Image size is $2.5 \times 2.5$ degrees. The third and fourth images display deformed star profiles as during this time $(\operatorname{since} T+24 \mathrm{~s}$ till $T+31 \mathrm{~s})$ REM robotic telescope (which has TORTORA camera mounted on top) repointed after receiving the burst information from Swift. Initially, burst position was on the edge of field of view, as a result of repointing it moved to the center of field of view, which resulted in better data quality.

data and the correspondingly rebinned gamma-ray one is no more than 0.5 , due to high level of stochastic component in 0.1-1 s range in both optical (measurements noise) and gamma rays (actual high-frequency variability) [39]. For the low-resolution data, with a $1.3 \mathrm{~s}$ binning, the correlation coefficient is, however, as high as 0.82 if the optical light curve is shifted 2 seconds back with respect to gamma-ray one (see Figure 7). Correspondingly rebinned gamma-ray data demonstrate the same four nearly equidistant peaks as optical ones.

This is the first detection of a close relation between the temporal structures of the optical and gamma-ray prompt emission. In our case, the gamma-ray burst itself precedes the optical flash by two seconds. This feature, along with the periodicities we detected, have a serious physical implications for the models of the event, as they clearly contradict most of proposed variants of emission generation [26]. They are, however, inevitably suggest the periodic behaviour of the internal engine, which may be explained by the onset of instabilities and the gravimagnetic precession of the massive accretion disk around the newborn stellar-mass black hole.

\section{MegaTORTORA}

It is important to develop the methodology of wide-field search for fast optical transients in two directions. The first is the increase of detection threshold by 2-3 magnitudes while keeping the field of view and temporal resolution. It may be achieved by means of multiobjective (or multitelescope) systems, by decreasing field of view of single instrument and, therefore, its pixel scale [40]. To avoid the dominance of CCD read-out noise, the quantum efficiency and amplification of image intensifier have to be increased, or the low-noise fast EM-CCDs may be used instead. The second direction is the acquisition of the spectral, or at least multicolor, and polarimetric information for the transients.

One possible design of a multiobjective monitoring system with EM-CCDs, able to collect multicolor and polarimetric information, is presented below.

5.1. Basic $3 \times 3$ Unit. The project utilizes the modular design and consists of a set of basic units, 9 objectives each, installed on a separate mounts (see Figure 8). Each 


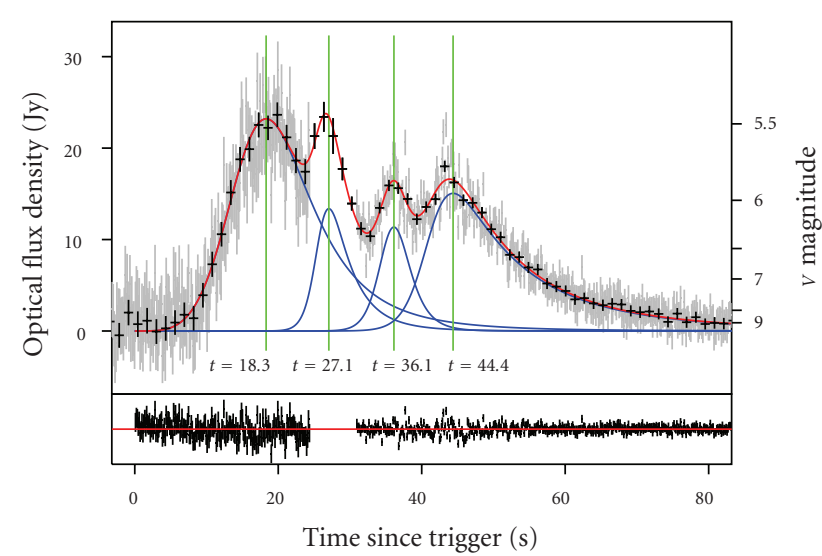

FIgURE 5: The light curve of GRB080319B acquired by TORTORA wide-field camera. The gamma-emission started at $T \approx-4 \mathrm{~s}$ and faded at $T \approx 55 \mathrm{~s}$. Full resolution ( $0.13 \mathrm{~s}$ exposure, gray lines) data are available for all duration of gamma-emission except for interval of REM telescope repointing $(24.5 \mathrm{~s}<T<31 \mathrm{~s})$, while lowresolution ones (summation of 10 consecutive frames, $1.3 \mathrm{~s}$ effective exposure) - for the whole time. The light curve is approximated by a four nearly equidistant flares; lower panel shows the residuals of such approximation.

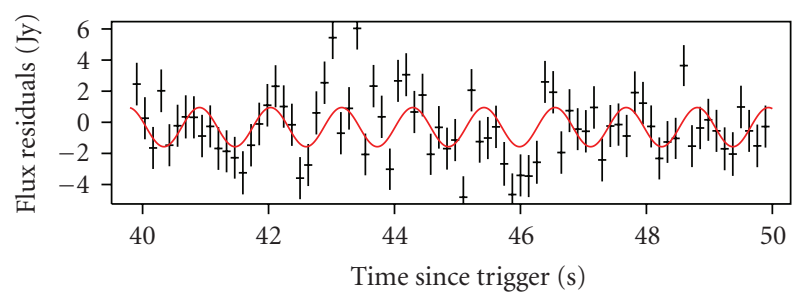

(a)

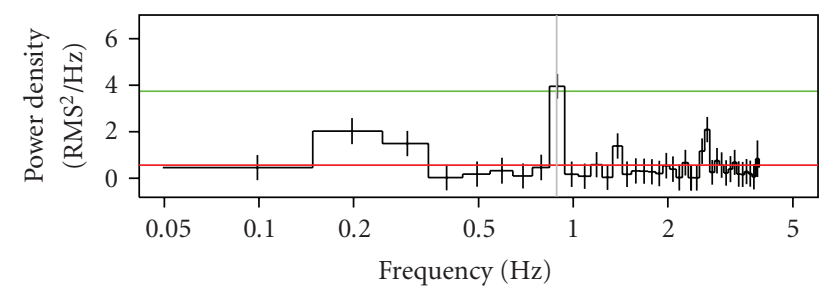

(b)

FIGURE 6: (a) Optical flux for a $T+40 s-T+50 s$ interval (last peak) with the approximation shown in Figure 5 subtracted. Smooth line shows the best-fit sinusoidal approximation of the data with $P=$ 1.13 s period. (b) Power density spectrum of this data, estimated by bootstrapping method-by generating a large number of sample time series by randomly shuffling the original light curve, what completely destroys its time structure while keeping the distribution of its values, and by studying the distribution and quantiles of resulting power densities. Horizontal lines represent mean noise level (lower) and a level of noise deviations with $10^{-3}$ significance (upper), estimated by bootstrapping number of time series from the original data set. Vertical line corresponds to the period of the sinusoidal approximation shown in (a), clearly coincided with the peak of power spectrum. The probability of a random appearance of a feature like the one seen in any of 39 frequency bin is $\sim 0.01$.

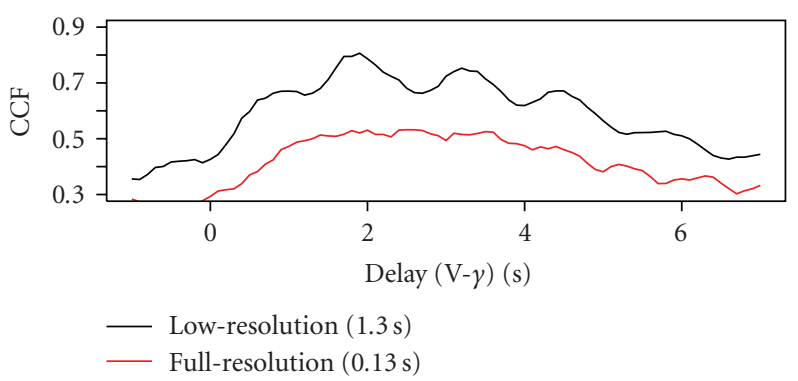

(a)

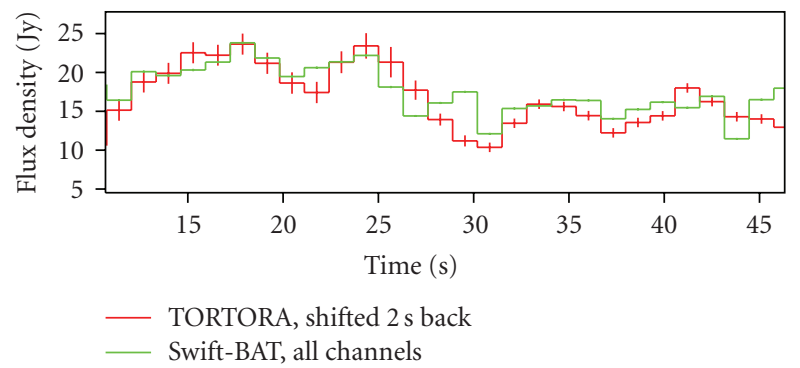

(b)

Figure 7: (a) Cross-correlation of the Swift-BAT gamma-ray (all energy channels) and TORTORA optical fluxes for the main (plateau) phase of the burst emission. (b) TORTORA optical flux shifted back 2 seconds along with correspondingly rebinned SwiftBAT gamma-ray flux. The correlation is $r=0.82$ with significance level of $5 \cdot 10^{-7}$. Gamma-ray curve is arbitrarily scaled and shifted for illustrative purposes.

objective in a unit is placed inside the gimbal suspension with remotelycontrolled micromotors, and so may be oriented independently from others. Also, each objective possesses the set of color and polarization filters, which may be installed before the objective on the fly. It allows to change modes of observation on the fly, from routine wide-field monitoring in white light, with no filters installed, to the narrow-field follow-up regime, when all objectives are pointed towards the same point, that is, newlydiscovered transient, and observe it in different colors and for different polarization plane orientations simultaneously, to acquire all possible kinds of information for the transient (see Figure 9). Simultaneous observation of the transient by all objectives in white light is also possible to get better photometric accuracy by coadding frames.

Each objective is equipped with the fast EM-CCD, which has a low readout noise even for a high frame rates when the internal amplification is in effect. Possible variants of a readilyavailable commercial EM-CCDs and objectives are shown in Figure 10.

The data from each channel of such a system, which is roughly 20 megabytes per second, is collected by a dedicated rackmount PC, which stores it in its hard-drive as well as performs its real-time data processing in a way similar to the current processing pipeline of FAVOR and TORTORA cameras, which currently operate under similar data flow rate. The whole system is coordinated by the central server which acquires the transient data from data-processing PCs 


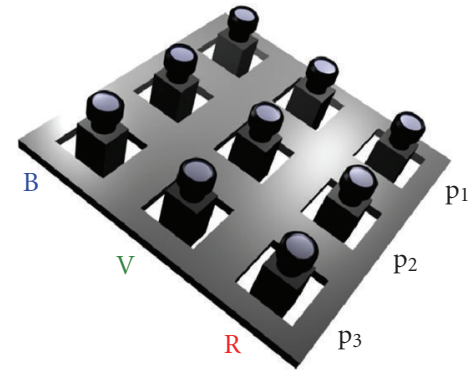

(a)

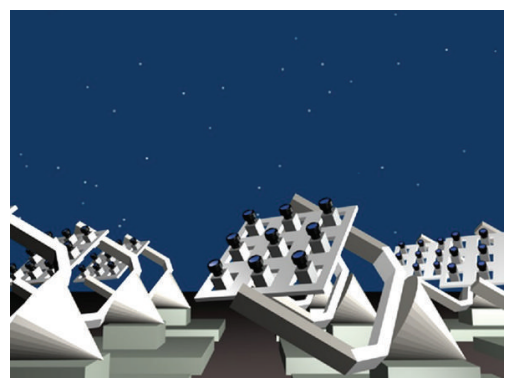

(b)

FIGURE 8: (a) Basic $3 \times 3$ objective unit. Each objective is able to repoint independently and has installable color and polarization filters. (b) The artistic view of a complete MegaTORTORA system, consisting of a number of basic units on separate equatorial mounts.

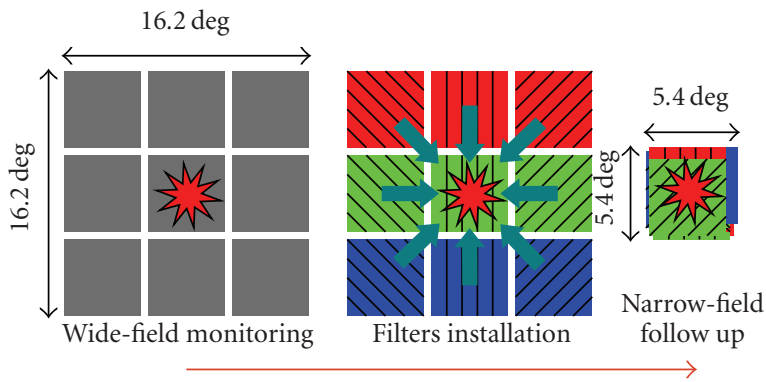

FIGURE 9: Different modes of operation of MegaTORTORA system. Left: wide-field monitoring mode in a white light. Middle: insertion of color and polarization filters as a first step of follow-up routine upon detection of a transient. Right: repointing of all unit objectives towards the transient. In the latter regime of operation the system collects three-color transient photometry for three polarization plane orientations (illustrated by a different stroke directions in figure) simultaneously. Mode transition speed depends on exact hardware parameters (objective and CCD weight, motors used, etc.), but is expected to be less than 0.3 seconds.

and controls the pointing and mode of operation of all objectives in response to them.

Each basic $3 \times 3$ unit in wide-field monitoring mode has $\sim 260$ square degrees field of view and has a $\sim 14.5^{\mathrm{m}}$ limit in a white light for a $0.1 \mathrm{~s}$ exposure if a sky background noise prevails over the read-out noise (i.e., in a high gain regime of EM-CCD). Frame coaddition can improve it up to $17^{\mathrm{m}}$ for effective exposure of $10 \mathrm{~s}$ and up to $19.5^{\mathrm{m}}$-for 1000 s. In narrow-field follow-up mode, with $\sim 30$ square degrees field, the limits depend on the selection of color filters and polarizers and are summarized in Table 4. Also, for a bright transients, a very high temporal resolution mode is possible, if the CCD supports the read-out in a small window with greater frame rate (e.g., Andor iXon ${ }^{\mathrm{EM}}+888$ EM-CCD provides the frame rates up to 65 in $128 \times 128$ window without binning, and up to 310 -with $8 \times 8$ binning).

5.2. Complete System. The complete system is a set of basic $3 \times 3$ units installed on a separate mounts and operated in
TABle 4: Detection limits (in stellar magnitudes) of a basic $3 \times 3$ unit in narrow-field follow-up mode for a different combinations of color and polarimetric filters in use.

\begin{tabular}{lcccc}
\hline Time scale, s & White/B & $\begin{array}{c}\text { B }+3 \\
\text { polarizations }\end{array}$ & BVR & $\begin{array}{c}\text { BVR }+3 \\
\text { polarizations }\end{array}$ \\
\hline 0.1 & 15.7 & 13.0 & 15.0 & 12.5 \\
10 & 18.2 & 15.2 & 17.5 & 15.0 \\
1000 & 20.7 & 17.9 & 20.0 & 17.5 \\
\hline
\end{tabular}

parallel. The number of units may be arbitrary-the larger the better.

As an example, let us assume 8 unit configuration. It will cover 2100 square degrees of the sky simultaneously in a wide-field monitoring mode, which allows to perform the all sky survey twice per night while staying for half an hour on each region. In a narrow-field mode, by combining the data from all 72 objectives, it will reach $17.2^{\mathrm{m}}$ to $19.7^{\mathrm{m}}$ limit for 0.1 to $10 \mathrm{~s}$ effective exposures. The amount of data acquired in a night of observations will be around $40 \mathrm{~Tb}$, which will be processed in real time. Such a system is expected to see the light of a GRB once per month. Performance of such a system for observations of different classes of variable objects is shown in Figure 11 in comparison with other wide-field monitoring projects.

As for financial side, assuming the prices as $2 \mathrm{kE}$ for objective, $45 \mathrm{kE}$ for EM-CCD, $1 \mathrm{kE}$ for data-processing PC and $26 \mathrm{kE}$ for an equatorial mount, a single basic $3 \times$ 3 unit will costs approximately $500 \mathrm{kE}$, while the 8 units configuration—approximately 5 millions of Euros.

\section{Conclusions}

The Naked-Eye Burst has stressed the importance of both wide-field monitoring and high temporal resolution for the search for and investigation of short optical transients of unknown localization. FAVOR and TORTORA cameras, created by our group, achieved significant results in this area, 


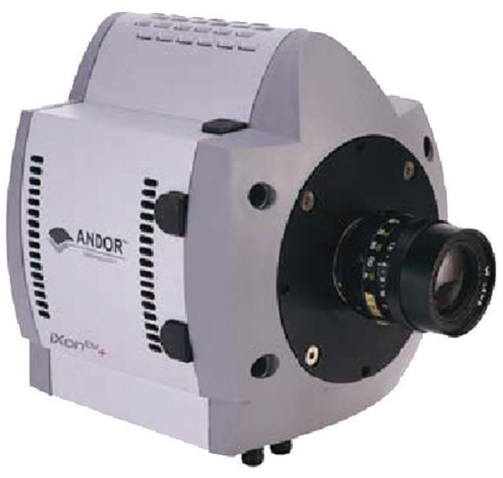

(a)

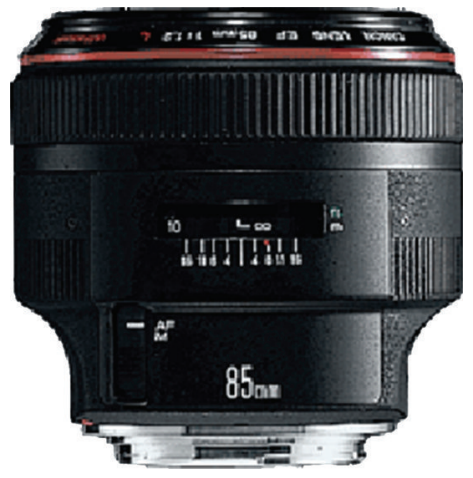

(b)

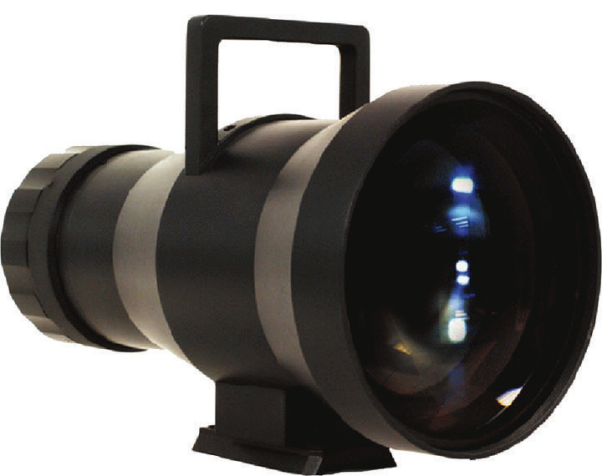

(c)

Figure 10: Possible commerciallyavailable parts for MegaTORTORA. (a) Andor iXon ${ }^{\mathrm{EM}}+8881024 \times 1024$ EM-CCD with $13 \mu \mathrm{m}$ pixel size, up to $95 \%$ quantum efficiency, 9 frames per second frame rate, and less than $1 \mathrm{e}^{-}$read-out noise in high gain regime. (b) Canon $\mathrm{EF} 85 \mathrm{f} / 1.2 \mathrm{~L}$ USM II objective, which may provide $9 \times 9$ degree field of view with $31^{\prime \prime}$ pixel scale for this CCD. (c) Marshall Electronics $140 \mathrm{~mm} \mathrm{f} / 1.0$ lens, which will give $5.4 \times 5.4$ degree field with $19^{\prime \prime}$ pixel scale.

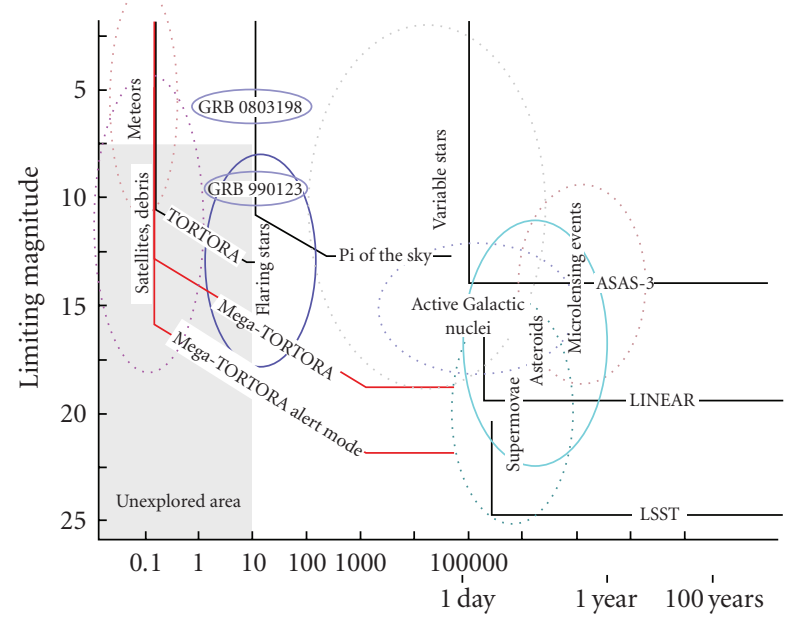

Temporal resolution (seconds)

Figure 11: Performance of MegaTORTORA, as well as several others typical wide-field monitoring projects both in operation now (ASAS-3, LINEAR, Pi of the Sky, FAVOR/TORTORA) and planned for near future (LSST), in observations of different classes of variable objects.

which allows us to propose the development of such strategy, and formulate the design of a next generation of a wide-field monitoring system—the MegaTORTORA.

\section{Acknowledgments}

This work was supported by the Bologna University Progetti Pluriennali 2003, by grants of CRDF (no. RP1-2394-MO02), RFBR (nos. 04-02-17555, 06-02-08313, and 09-0212053), INTAS (04-78-7366), and by the Presidium of the Russian Academy of Sciences Program. S. Karpov has also been supported by a grant from the President of Russian Federation for federal support of young scientists.

\section{References}

[1] S. McBreen, F. Quilligan, B. McBreen, L. Hanlon, and D. Watson, "Temporal properties of the short gamma-ray bursts," Astronomy and Astrophysics, vol. 380, no. 2, pp. L31-L34, 2001.

[2] S. Karpov, G. Beskin, A. Biryukov, et al., "Optical camera with high temporal resolution to search for transients in the wide field," Nuovo Cimento della Societa Italiana di Fisica C, vol. 28, no. 4-5, pp. 747-750, 2005.

[3] A. Piccioni, C. Bartolini, C. Cosentino, et al., "An updating about flip: a photometer devoted to the search for optical flashes from gamma-ray bursters ," in AIP Conference Proceedings, first-name middle-name M. Friedlander, N. Gehrels, and D. J. Macomb, Eds., vol. 280 of American Institute of Physics Conference Series, pp. 1152-1155, 1993.

[4] B. Paczyński, "Monitoring all sky for variability," Publications of the Astronomical Society of the Pacific, vol. 112, no. 776, pp. 1281-1283, 2000.

[5] S. D. Barthelmy, "Observing strategies using GCN," in Proceedings of the 4th Hunstville Symposium on Gamma-Ray Bursts, C. A. Meegan, R. D. Preece, and T. M. Koshut, Eds., vol. 428 of AIP Conference Proceedings, pp. 129-133, 1998.

[6] K. L. Page, R. Willingale, J. P. Osborne, et al., "GRB 061121: broadband spectral evolution through the prompt and afterglow phases of a bright burst," Astrophysical Journal, vol. 663, no. 2 I, pp. 1125-1138, 2007.

[7] S. A. Yost, H. F. Swan, E. S. Rykoff, et al., "Exploring broadband GRB behavior during $\gamma$-ray emission," Astrophysical Journal, vol. 657, no. 2 I, pp. 925-941, 2007.

[8] W. T. Vestrand, P. R. Wozniak, J. A. Wren, et al., "A link between prompt optical and prompt $\gamma$-ray emission in $\gamma$-ray bursts," Nature, vol. 435, no. 7039, pp. 178-180, 2005.

[9] C. Akerlof, R. Balsano, S. Barthelmy, et al., "Observation of contemporaneous optical radiation from a $\gamma$-ray burst," Nature, vol. 398, no. 6726, pp. 400-402, 1999.

[10] T. Tamagawa, F. Usui, Y. Urata, et al., "The search for optical emission on and before the GRB trigger with the WIDGET 
telescope," Nuovo Cimento della Societa Italiana di Fisica C, vol. 28, no. 4-5, pp. 771-774, 2005.

[11] K. Borozdin, S. Brumby, M. Galassi, et al., "Real-time detection of optical transients with RAPTOR," in Astronomical data Analysis II, vol. 4847 of Proceedings of SPIE, pp. 344-353, Waikoloa, Hawaii, USA, August 2002.

[12] A. J. Castro-Tirado, J. Soldán, M. Bernas, et al., "The burst observer and optical transient exploring system (BOOTES)," Astronomy and Astrophysics Supplement Series, vol. 138, no. 3, pp. 583-585, 1999.

[13] A. J. C. Tirado, M. Jelínek, S. Vítek, et al., "A very sensitive allsky CCD camera for continuous recording of the night sky," in Advanced Software and Control for Astronomy II, vol. 7019 of Proceedings of SPIE, Marseille, France, June 2008.

[14] A. Burd, M. Cwiok, H. Czyrkowski, et al., "Pi of the skyall-sky, real-time search for fast optical transients," New Astronomy, vol. 10, no. 5, pp. 409-416, 2005.

[15] D. Eichler, M. Livio, T. Piran, and D. N. Schramm, "Nucleosynthesis, neutrino bursts and $\gamma$-rays from coalescing neutron stars," Nature, vol. 340, no. 6229, pp. 126-128, 1989.

[16] S. E. Woosley, "Gamma-ray bursts from stellar mass accretion disks around black holes," Astrophysical Journal, vol. 405, no. 1, pp. 273-277, 1993.

[17] B. Paczyński, "Are gamma-ray bursts in star-forming regions?" Astrophysical Journal, vol. 494, no. 1, part 2, pp. L45-L48, 1998.

[18] G. M. Beskin, V. Plokhotnichenko, C. Bartolini, et al., "Catching the light curve of flaring GRBs: the opportunity offered by scanning telescopes," Astronomy and Astrophysics Supplement Series, vol. 138, no. 3, pp. 589-590, 1999.

[19] D. Eichler and G. Beskin, "Optical search for extraterrestrial intelligence with air Cerenkov telescopes," Astrobiology, vol. 1, no. 4, pp. 489-493, 2001.

[20] I. Zolotukhin, G. Beskin, A. Biryukov, et al., "Optical camera with high temporal resolution to search for transients in the wide field," Astronomische Nachrichten, vol. 325, no. 6-8, p. 675, 2004.

[21] F. Zerbi, G. Chincarini, M. Rodonò, et al., "REM-Rapid Eye Mount. A fast slewing robotized telescope to monitor the prompt infrared afterglow of GRBs," in Proceedings of the 2nd Workshop on Gamma-Ray Bursts in the Afterglow Era, E. Costa, F. Frontera, and J. Hjorth, Eds., p. 434, 2001.

[22] E. Molinari, S. Bondar, S. Karpov, et al., "TORTOREM: twotelescope complex for detection and investigation of optical transients," Nuovo Cimento della Societa Italiana di Fisica B, vol. 121, no. 12, pp. 1525-1526, 2006.

[23] G. Beskin, V. Bad'in, A. Biryukov, et al., "FAVOR (FAst Variability Optical Registration)—a two-telescope complex for detection and investigation of short optical transients," Nuovo Cimento della Societa Italiana di Fisica C, vol. 28, no. 4-5, pp. 751-754, 2005.

[24] S. Karpov, G. Beskin, S. Bondar, et al., "GRB 080319B: RAPTOR observations of a naked eye burst," in GRB Coordinates Network Circular, vol. 7452, 2008.

[25] J. L. Racusin, S. V. Karpov, M. Sokolowski, et al., "Broadband observations of the naked-eye $\gamma$-ray burst GRB 080319B," Nature, vol. 455, no. 7210, pp. 183-188, 2008.

[26] G. Beskin, S. Karpov, S. Bondar, et al., "Fast optical variability of Naked-Eye Burst-manifestation of periodic activity of internal engine," http://arxiv.org/abs/0905.4431.

[27] S. Karpov, G. Beskin, S. Bondar, et al., "Monitoring the sky in sub-second domain," Advances in Astronomy, vol. 2010, Article ID 784141, 8 pages, 2010.
[28] American Department of Defense satellite ephemerides database, http://www.space-track.org/.

[29] E. Bertin and S. Arnouts, "SExtractor: software for source extraction," Astronomy \& Astrophysics, vol. 117, no. 2, pp. 393404, 1996.

[30] E. Høg, C. Fabricius, V. V. Makarov, et al., "The Tycho-2 Catalogue of the 2.5 million brightest stars," Astronomy \& Astrophysics, vol. 363, no. 1, pp. L27-L30, 2000.

[31] A. Guarnieri, C. Bartolini, G. Beskin, et al., "GRB 060719: TORTOREM optical upper limits," GRB Coordinates Network, Circular Service, 5372, 1, 2006.

[32] S. Karpov, G. Beskin, S. Bondar, et al., "GRB 061202: TORTOREM optical upper limits," GRB Coordinates Network, Circular Service, 5897, 1, 2006.

[33] S. Karpov, G. Beskin, S. Bondar, et al., "GRB 061218: TORTOREM optical upper limits," GRB Coordinates Network, Circular Service, 5941, 1, 2006.

[34] J. L. Racusin, N. Gehrels, S. T. Holland, et al., GRB Coordinates Network Circular, 7427, 1, 2008.

[35] M. Cwiok, W. Dominik, G. Kasprowicz, et al., "GRB 080319b prompt optical observation by Pi-of-the-Sky," GRB Coordinates Network, Circular Service, 7439, 1, 2008.

[36] P. Wozniak, W. T. Vestrand, J. Wren, and H. Davis, "GRB 080319B: RAPTOR observations of a naked eye burst," GRB Coordinates Network, Circular Service, 7464, 1, 2008.

[37] D. Kocevski, F. Ryde, and E. Liang, "Search for relativistic curvature effects in gamma-ray burst pulses," Astrophysical Journal, vol. 596, no. 1, pp. 389-400, 2003.

[38] G. Beskin, S. Karpov, S. Bondar, et al., "TORTORA discovery of naked-eye burst fast optical variability," in Nanjing GammaRay Burst Conference, Y.-F. Huang, Z.-G. Dai, and B. Zhang, Eds., vol. 1065 of AIP Conference Proceedings, pp. 251-254, 2008.

[39] R. Margutti, C. Guidorzi, G. Chincarini, F. Pasotti, S. Covino, and J. Mao, "Temporal variability of GRB early X-ray afterglows and GRB080319B prompt emission," in Nanjing Gamma-Ray Burst Conference, Y.-F. Huang, Z.-G. Dai, and B. Zhang, Eds., vol. 1065 of AIP Conference Proceedings, pp. 259262, 2008.

[40] G. Beskin, V. de-Bur, S. Karpov, et al., "Search for optical signals from extra-terrestrial intelligence at SAO RAS: past, present and future," Bulletin of Special Astrophysical Obervatory, vol. 60-61, pp. 217-225, 2007. 

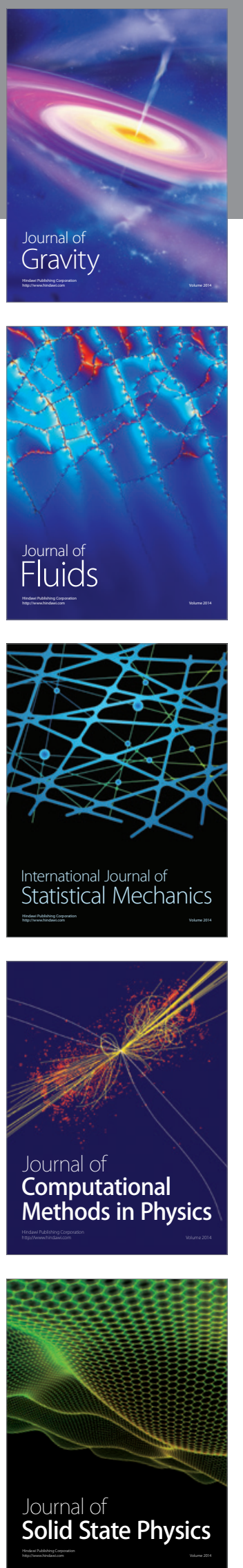

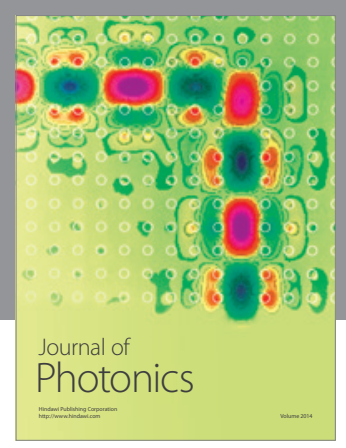

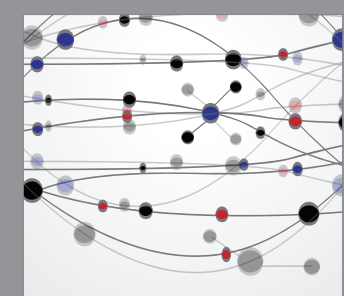

The Scientific World Journal
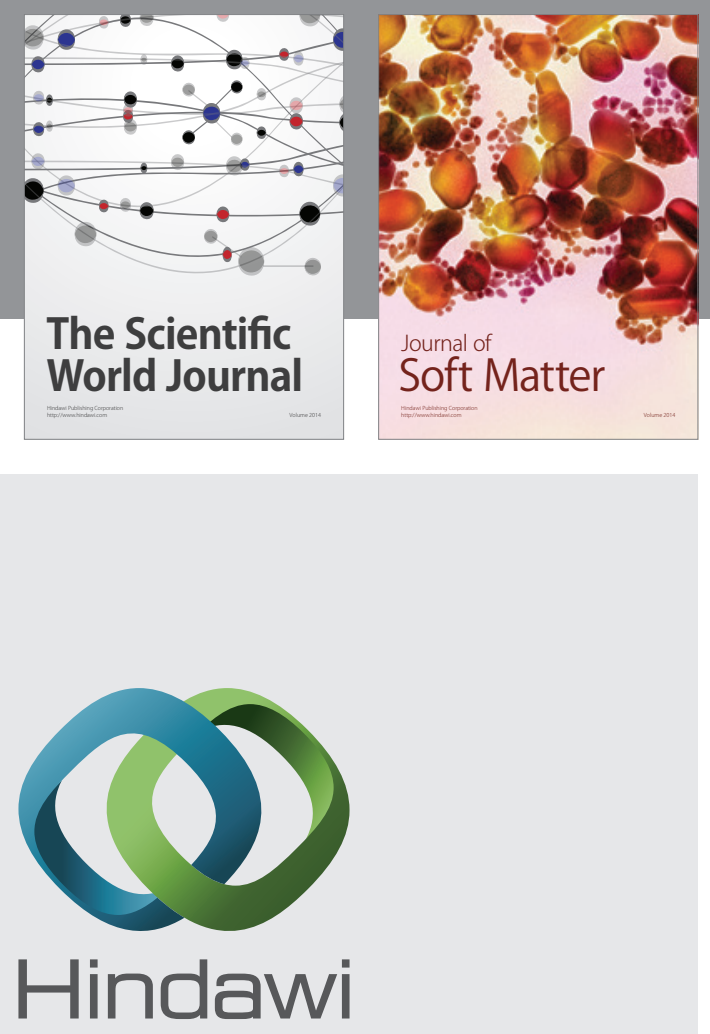

Submit your manuscripts at

http://www.hindawi.com
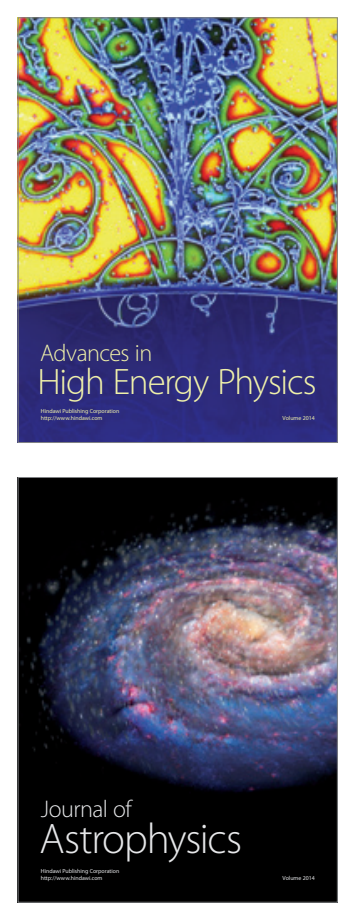
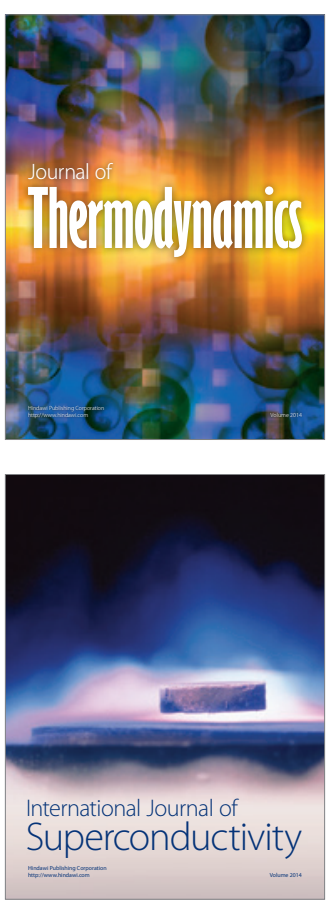
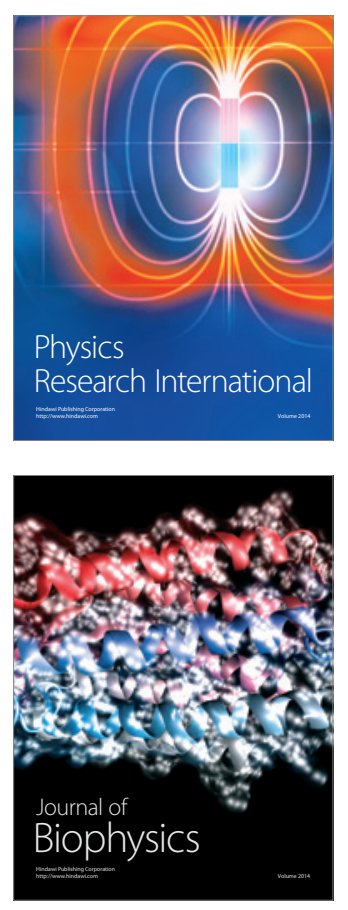
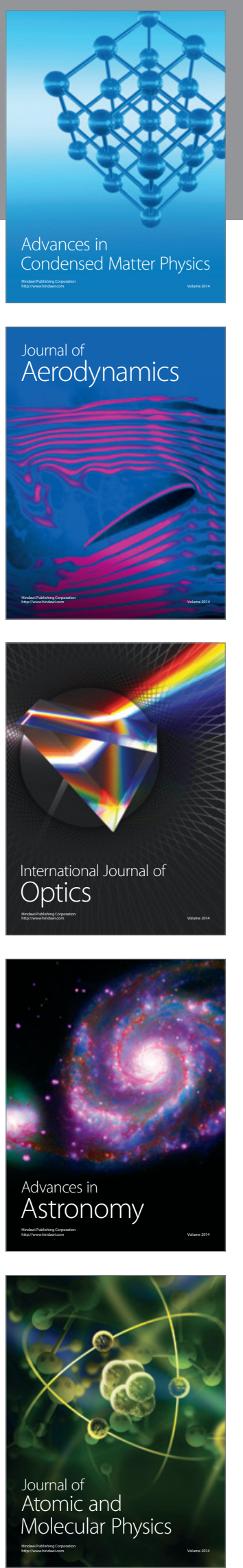